quenz betrug etwa 40/min. Die isolierte Wärmung des Rückenmarks von der 10. bis zur $20 \mathrm{~min}$ (Wassertemperatur $46^{\circ} \mathrm{C}$ ) führte zu einer sprunghaften Steigerung der Atemfrequenz auf 150 bis $200 / \mathrm{min}$, wobei das Tier das typische Bild des Hechelns mit Öffnung des Mauls, heraushängender Zunge und deutlicher Salivation bot. Während die im Wirbelkanal gemessene Temperatur auf $39,2^{\circ} \mathrm{C}$ stieg, sanken Rectal- und

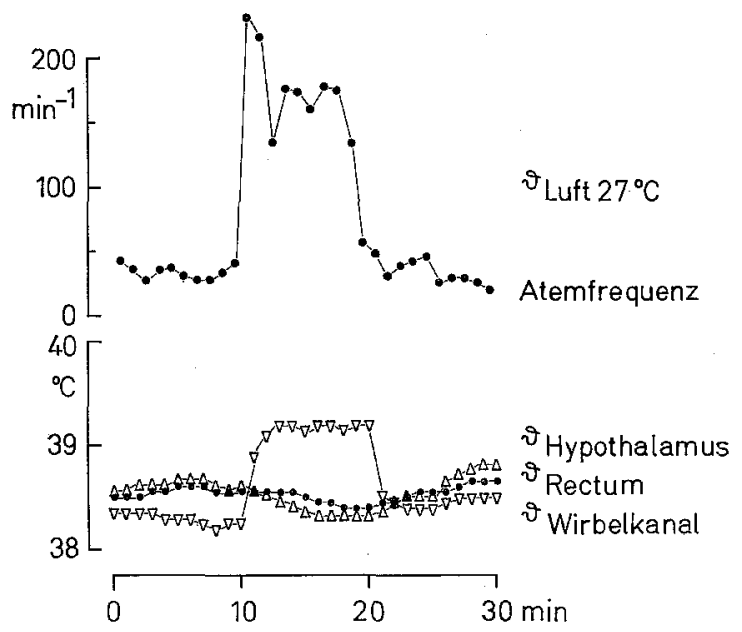

Fjg, 1. Isolierte Wärmung des Rückenmarks am wachen Hund mittels periduraler Thermoden von der 10. bis zur 20. min der Versuchsperiode. Lufttemperatur $27^{\circ} \mathrm{C}$, Wassertemperatur $46^{\circ} \mathrm{C}$, Hund $10 \mathrm{~kg}$, dünnes Fell

Hypothalamustemperatur als Folge der erhöhten Wärmeabgabe nach außen um $0,2^{\circ} \mathrm{C}$. Im Verlauf der Nachperiode stellten sich Kerntemperaturen und Atemfrequenz wiederum auf die Ausgangswerte ein. Die in dieser Weise vorgenommenen Wärmungen des Rückenmarks führten bei zwei Hunden in 23 Versuchen mit 54 Wärmperioden regelmäßig zu einer Steigerung der Atemfrequenz. In 20 Wärmperioden konnte typisches Hecheln ausgelöst werden.

Die Ergebnisse zeigen, daß die Wärmung des Rückenmarks am wachen Hund die Atmung in einer thermoregulatorisch sinnvollen Weise umstellen kann. Das legt den Schluß nahe, daß die durch die Wärmung des Rückenmarks hervorgerufene Erregung auch in höheren Abschnitten des Zentralnervensystems verarbeitet wird.

Eingegangen am 23. Januar 1967

[1] Simon, E., W. Rautenberg, R. Thauer u. M. Iriki: Naturwissenschaften 50, 337 (1963). - [2] JEsSEN, C., K.-A. MEURER u. E. Simon: Pflüger's Arch. ges. Physiol. 291, R 76 (1966). [3] Simon, E., W. Rautengerg, R. Thauer 1. M. Iriki: Pflüger's Arch. ges. Physiol. 281, 309 (1964).

\section{Fehlender Sexualthythmus bei Karpfen in Warmwasserhaltung}

Ch. Meske, B. LüHr und W. Szablewski

Max-Planck-Institut für Kulturpflanzenzüchtung, Hamburg-Volksdorf

Nachdem es uns gelungen war, den sog. Raumfaktor bei der Aufzucht von Karpfen (Cyprinus carpio) in engen Gefäßen zu überwinden und auch auf kleinstem Raum (Aquarien) besten Zuwachs zu erzielen [1-3]), galt es für züchterische Arbeiten die Geschlechtsreife schneller als unter Freilandbedingungen zu erreichen. Wir konnten die Geschlechtsreife bereits bei zweijährigen Karpfen beobachten, die mit einem Anfangsgewicht von $10 \mathrm{~g}$ mit 6 Monaten in die Aquarienhaltung bei ständig $23^{\circ} \mathrm{C}$ Wassertemperatur genommen worden waren. (Im Freiland werden die Karpfen ab 4. Lebensjahr geschlechtsreif.) Von diesen zweijährigen Tieren konnten durch Hypophyseninjektion Rogen und Milch gewonnen werden und die befruchteten Eier erbrütet werden. Das Ablaichen wurde im August erzielt, also außerhalb der normalen Laichzeit (Mai, Juni).
Wir konnten jetzt, im Januar, ebenfalls durch Hypophysierung das erneute Ablaichen der gleichen Elterntiere erreichen, also nur fünf Monate später. Nach dem Abstreifen der männlichen und weiblichen Geschlechtsprodukte wurde die $\mathrm{Be}$ fruchtung der Eier in Schalen vollzogen. Nach Behandlung mit einer Carbamid-NaCl-Lösung und anschließendem Waschen mit Tannin folgte das Erbrüten in Zuger-Gläsern unter ständigem Wasserdurchfluß (Methode nach Woynarovich [4]). Nach 4 Tagen schlüpften aus $90 \%$ der Eier die Jungfische.

Diese Ergebnisse zeigen: 1. In Verbindung mit der von uns entwickelten Warmwasserhaltung ist es bei ganzjährigem $\mathrm{Zu}$ wachs möglich, die Geschlechtsreife von Karpfen in der halben Zeit zu erreichen wie im Freiland. Die Generationenfolge läßt sich also erheblich beschleunigen, wodurch züchterische Arbeiten schneller als bisher möglich sind. 2. Das Ablaichen von Aquarienkarpfen ist mehrmals im Jahr möglich, mindestens alle 5 Monate. 3. Es besteht kein endogener Sexualrhythmus bei Karpfen. Bei konstanten Umweltbedingungen lassen sich reife Geschlechtsprodukte zu jeder Jahreszeit gewinnen. 4. Das Verfahren ermöglicht die gezielte Züchtung durch Paarung von Einzelindividuen, ,in vitro“"

Eingegangen am 3. Februar 1967

[1] Sengbusch, R. v., Ch. Meske u. W. Szablewski: Experientia 21, 614 (1965. - [2] Sengbusch, R. v., B. Lühr, Ch. Meske u. W. Szablews i : Arch. Fischereiwiss. 17, 89 (1966). - [2] SENGBUsch, R. v., Ch. Meske, W. Szablewski u. B. LÜhr: Z. Fischerei 1967 (im Druck). - [4] Woynarovich, E.: Allg. Fischerei-Ztg. 86, Nr. 22 (1961).

\section{Unterschiedliche Geschwindigkeiten leichter und schwerer Spermien im Ejakulat}

E. ScHILIING und P. Schmid

Max-Planck-Institụt für Tierzucht und Tierernährung, Mariensee

und Institut für Tierzucht ETH, Zürich

Durch ein bestimmtes Sedimentationsverfahren ist es möglich, die Samenzellen von Bullen in leichte und schwere Spermien zu trennen. Besamungen mit schweren Samenzellen führten zu einem höheren Anteil an weiblichen Kälbern $[1,2]$. Vermutlich handelt es sich bei diesen schweren Samenzellen um X-Spermien, bei den leichten um Y-Spermien. Mögliche Unterschiede der Geschwindigkeiten zwischen schweren und leichten Spermien wurden jetzt beim Schaf überprüft. Messungen an den Spermienköpfen ergaben, daß die der leichten Spermien $4,8 \%$ kürzer und $6,7 \%$ schmaler waren. Die Vorwärtsbewegung wurde nach zwei Methoden bestimmt. Bei dem indirekten Verfahren nach RoTHschiLd [3] mißt man die durchschnittliche Geschwindigkeit einer Spermiensuspension auf kinematographischem Wege. Die Berechnung stützt sich auf die Differenz in der Spermienzahl der sich in bestimmten Zeitabständen folgenden Bilder $(8,4,2 / \mathrm{sec})$. Bei zwei Proben wurde die Spermiengeschwindigkeit zudem auch direkt gemessen, und zwar durch Aufzeichnen der Bewegungsbahnen von jeweils 30 bis 35 Samenzellen aus denselben Filmaufnahmen, wie sie für die indirekte Methode benutzt wurden (16 Bilder/sec). Leichte und schwere Spermienfraktionen aus gleichen Ejakulaten wurden gleich vorbehandelt; sie wurden nach der Trennung zentrifugiert und mit einer Citrat-Gykokoll-Lösung aufgenommen. Gleichgroße Tropfen wurden auf denselben Objektträger gebracht und bei $37^{\circ} \mathrm{C}$ abwechselnd gefilmt. Die Geschwindigkeitsmessungen erfolgten jeweils $1 \mathrm{~h}$ und $12 \mathrm{~h}$ nach der Trennung.

\section{Ergebnisse}

Mit der indirekten Methode wurde in neun Trennungsversuchen festgestellt, daß alle Proben mit schweren Samenzellen geringere Durchschnittsgeschwindigkeiten aufwiesen. Die Differenzwerte lagen zwischen 12,3 bis $55 \%$ geringerer Vorwärtsbewegung: Im Durchschnitt aller Proben wurde eine um $32,4 \%$ verminderte Vorwärtsbewegung festgestellt. Die absoluten Zahlen lagen bei $187 \mu / \mathrm{sec}$ für die leichten Spermien und bei $124 \mu /$ sec für die schweren Spermien $(p<0,1 \%)$. Bei der direkten Messung der Bewegungsbahnen von Spermien pro Zeiteinheit wurde in zwei Versuchen ermittelt, daß die Vorwärtsbewegung der schweren Samenzellen um $28,6 \%$ und 